\title{
On errors of digital particle image velocimetry
}

\author{
H Huang, D Dabiri and M Gharib
}

Center of Quantitative Visualization, Graduate Aeronautical Laboratories of California Institute of Technology, Pasadena, CA 91125, USA

Received 12 June 1997, accepted for publication 24 September 1997

\begin{abstract}
The goal of the present study is to quantify and reduce, when possible, errors in two-dimensional digital particle image velocimetry (DPIV). Two major errors, namely the mean bias and root-mean-square (RMS) errors, have been studied. One fundamental source of these errors arises from the implementation of cross correlation (CC). Other major sources of these errors arise from the peak-finding scheme, which locates the correlation peak with a sub-pixel accuracy, and noise within the particle images. Two processing techniques are used to extract the particle displacements. First, a CC method utilizing the FFT algorithm for fast processing is implemented. Second, a particle image pattern matching (PIPM) technique, usually requiring a direct computation and therefore more time consuming, is used. Using DPIV on simulated images, both the mean-bias and RMS errors have been found to be of the order of 0.1 pixels for CC. The errors of PIPM are about an order of magnitude less than those of CC. In the present paper the authors introduce a peak-normalization method which reduces the error level of $\mathrm{CC}$ to that of PIPM without adding much computational effort. A

peak-compensation technique is also introduced to make the mean-bias error negligible in comparison with the RMS error. Noise in an image suppresses the mean-bias error but, on the other hand, significantly amplifies the RMS error. A digital video signal usually has a lower noise level than that of an analogue one and therefore provides a smaller error in DPIV.
\end{abstract}

\section{Introduction}

It is no longer necessary to stress the importance of particle image velocimetry (PIV) in modern fluid mechanics research. As a quantitative flow visualization tool, the recently emerging PIV has already been challenged in a wide range of applications (Grant 1994, 1997). The major advantage of PIV over the traditional techniques such as hot-wire anemometry (HWA) and laser-Doppler velocimetry (LDV) is its ability to provide instantaneous two-dimensional velocity fields.

One of the challenges in the application of PIV is to determine its error. The error of PIV depends on many factors. Owing to its complexity, error quantification is a function of the experimental conditions and the particledisplacement detection algorithms used. Researchers are making an effort to solve the problem piece by piece beginning with validation of the velocity. Velocity vectors obtained with poor correlations are considered invalid and are referred to as outliers. The source for the outliers can be from three-dimensional motion, large velocity gradients, improper particle seeding and so on. Detailed studies can be found in many previous publications (Keane and Adrian 1990, Willert 1992, Huang et al 1993a, Westerweel 1993).

Also of concern is the error of the valid velocities. Guezennec and Kiritsis (1990) first addressed this issue

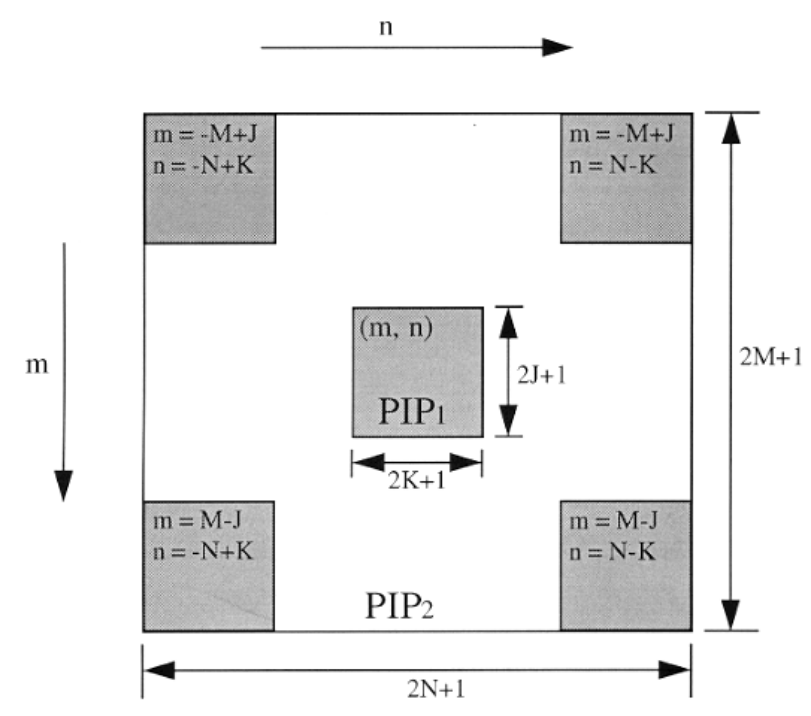

Figure 1. An illustration of PIP matching: $\mathcal{P} \mathcal{I} \mathcal{P}_{1}$ is confined inside $\mathcal{P} \mathcal{I P}_{2}$ and scans over $\mathcal{P} \mathcal{I P} \mathcal{P}_{2}$ from top-left to bottom-right.

by studying the PIV velocity error as a function of the image noise level, number of particles and particle displacement. Willert (1992) analysed the error as a 


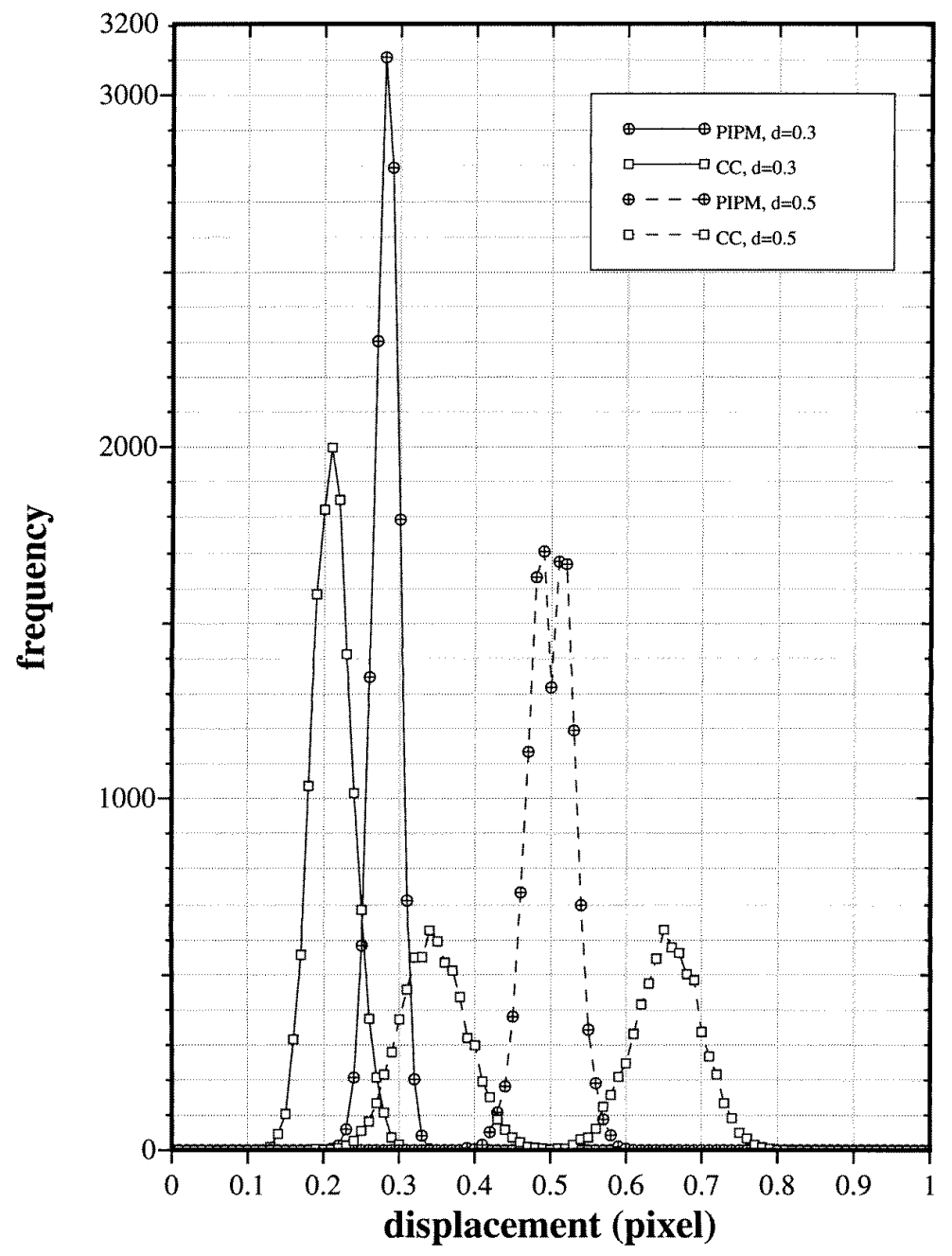

Figure 2. An example of the DPIV response to the input of unique displacements $(d=0.3$ and 0.5 pixels) applied to all particles for PIPM and cross correlation (CC).

function of the particle density, particle displacement and velocity gradients. Prasad et al (1992) investigated the effect of spatial resolution on the accuracy of velocity vectors. Lourenco and Krothapalli (1995) studied the effects of various correlation peak-finding schemes on the velocity errors. Boillot and Prasad (1996) recently provided an optimization procedure for pulse separation to minimize this error. Therefore, most of the published works have focused on the errors arising from the measured flow, experimental set-up and experimental conditions, whereas few have paid attention to the errors caused by the PIV processing method.

The present work, therefore, focuses on the error quantification associated with the processing method of the digital version of PIV (hereafter DPIV). New and improved processing techniques providing accurate correlation peak locations in a discrete correlation function are introduced and their errors are discussed. Error analyses are first made with noise-free simulated images. The parameters of these simulated images, such as the particle displacement, seeding density and so on, are known a priori and therefore errors solely arising from the processing method can be quantified. In order to have a close error estimation in the real experiment, the noise effect from the video camera is also examined.

\section{Digital PIV (DPIV)}

\subsection{Cross correlation (CC)}

In DPIV the particle displacement is usually determined by cross correlating small image masks of singly exposed image pairs. The discrete cross correlation function is defined as

$$
\mathcal{C}(m, n)=\sum_{i} \sum_{j} \mathcal{P} \mathcal{I P}_{1}(i, j) \mathcal{P} \mathcal{I P}_{2}(i-m, j-n)
$$

where $\mathcal{P} \mathcal{I} \mathcal{P}_{1}$ and $\mathcal{P} \mathcal{I} \mathcal{P}_{2}$ are the image masks in the first and second image of the singly exposed image pair, respectively. We call the image mask the particle image pattern (PIP). The maximum peak location in $\mathcal{C}(m, n)$ indicates the particle displacement.

Equation (1) can be computed either directly in the spatial domain or in the frequency domain via a fast fourier 
transform (FFT) algorithm (Gonzalez and Wintz 1987, Willert and Gharib 1991). The FFT algorithm provides a fast implementation of equation (1), especially when the size of $\mathcal{P} \mathcal{I} \mathcal{P}_{1}$ and that of $\mathcal{P} \mathcal{I} \mathcal{P}_{2}$ are the same. Thus, for the sake of fast processing, $\mathcal{P} \mathcal{I} \mathcal{P}_{1}$ and $\mathcal{P} \mathcal{I} \mathcal{P}_{2}$ are usually the same size in DPIV. As the particle displacement increases, however, the error of the $\mathrm{CC}$ location increases since the number of correlated particles remaining in $\mathcal{P} \mathcal{I P}_{2}$ decreases (Huang et al 1993a). This is referred to as the out-of-pattern effect. However, this error can be significantly reduced by the image shifting technique (Westerweel et al 1997).

Actually, the error caused by the out-of-pattern effect can be avoided by properly choosing the sizes of $\mathcal{P} \mathcal{I} \mathcal{P}_{2}$. Given $\mathcal{P} \mathcal{I} \mathcal{P}_{1}$ of size $(2 J+1) \times(2 K+1)$ and the maximum particle displacement $D$, enlarging $\mathcal{P} \mathcal{I} \mathcal{P}_{2}$ to the size of $(2 M+1) \times(2 N+1)$, which satisfies

$$
\min \{(M-J),(N-K)\}>D
$$

produces the correct correlation peak without shifting $\mathcal{P I P} \mathcal{P}_{1}$ to outside $\mathcal{P} \mathcal{I} \mathcal{P}_{2}$, as illustrated in figure 1. That means, all correlated particles will remain in $\mathcal{P} \mathcal{I P}_{2}$. The correlation under this special condition is referred to as PIP matching (PIPM) (Huang et al 1993a). Because of the difference between the sizes of $\mathcal{P} \mathcal{I} \mathcal{P}_{1}$ and $\mathcal{P} \mathcal{I} \mathcal{P}_{2}$, its FFT implementation is less efficient.

The cross correlation function given in equation (1), although simple in nature, has the drawback that it is sensitive to intensity changes in $\mathcal{P} \mathcal{I P} \mathcal{P}_{1}$ and $\mathcal{P} \mathcal{I} \mathcal{P}_{2}$. A method frequently used to overcome this difficulty is to normalize equation (1) as

$$
\begin{aligned}
\mathcal{R}(m, n) & =\left\{\sum_{i} \sum_{j}\left[\mathcal{P I P}_{1}(i, j)-\overline{\mathcal{P} \mathcal{I P}_{1}}\right]\right. \\
& \left.\times\left[\mathcal{P I}_{\mathcal{I}}(i-m, j-n)-\overline{\mathcal{P} \mathcal{I P}_{2}}\right]\right\} \\
& \times\left\{\left[\sum_{i} \sum_{j}\left(\mathcal{P I P}_{1}(i, j)-\overline{\mathcal{P} \mathcal{I P}_{1}}\right)^{2}\right.\right. \\
& \left.\left.\times \sum_{i} \sum_{j}\left(\mathcal{P I P P}_{2}(i, j)-\overline{\mathcal{P} \mathcal{I} \mathcal{P}_{2}}\right)^{2}\right]^{1 / 2}\right\}^{-1}
\end{aligned}
$$

where $\overline{\mathcal{P} \mathcal{I} \mathcal{P}_{1}}$ and $\overline{\mathcal{P} \mathcal{I} \mathcal{P}_{2}}$ are the means of $\mathcal{P} \mathcal{I} \mathcal{P}_{1}$ and $\mathcal{P} \mathcal{I} \mathcal{P}_{2}$, respectively (Gonzalez and Wintz 1987). Equation (3) is considerably more difficult to implement using the FFT and is therefore computed directly in the spatial domain. The PIPM is implemented using the directly computed normalized correlation function given in equation (3) (Huang et al 1993a).

\subsection{Sub-pixel peak locating}

In PIV, the location of the correlation peak is directly associated with the particle displacement. Obviously, an error of \pm 0.5 pixels is present in the correlation peak location provided by the discrete correlation function given in equations (1) and (3). The maximum data point in a discrete correlation is an integer peak. To increase the PIV accuracy, it is necessary to locate the correlation peak more precisely; that is, to within a sub-pixel accuracy. Many

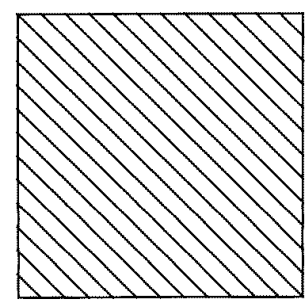

(a) PIP1

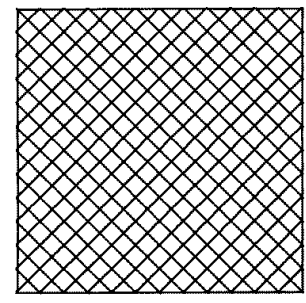

(c) $\mathrm{C}(0,0): \mathrm{PIP} 2$

fully over PIP1

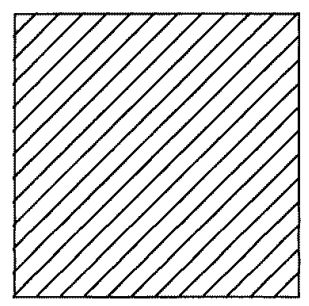

(b) $\mathrm{PIP} 2$
Figure 3. The mean-bias and RMS errors of PIPM and NCC as functions of the particle displacement.

methods, such as centre-of-mass, parabolic curve-fitting and Gaussian curve-fitting methods (Willert and Gharib 1991), have been used to obtain the correlation peak to within subpixel accuracy. For all of these methods, the correlation peak's location is a function of the shape of the correlation peak. Among them, the Gaussian curve-fitting method is frequently used in many DPIV implementations.

Suppose that an integer peak has been found at $\left(x_{0}, y_{0}\right)$ in a correlation function $\mathcal{R}(m, n)$. Assuming the correlation peak has a Gaussian-distribution shape, the accurate location of the correlation peak is

$$
\begin{aligned}
x= & x_{0}+\left\{\log \mathcal{R}\left(x_{0}-1, y_{0}\right)-\log \mathcal{R}\left(x_{0}+1, y_{0}\right)\right\} \\
& \times\left\{2 \left[\log \mathcal{R}\left(x_{0}-1, y_{0}\right)+\log \mathcal{R}\left(x_{0}+1, y_{0}\right)\right.\right. \\
& \left.\left.-2 \log \mathcal{R}\left(x_{0}, y_{0}\right)\right]\right\}^{-1} \\
y= & y_{0}+\left\{\log \mathcal{R}\left(x_{0}, y_{0}-1\right)-\log \mathcal{R}\left(x_{0}, y_{0}+1\right)\right\} \\
& \times\left\{2 \left[\log \mathcal{R}\left(x_{0}, y_{0}-1\right)+\log \mathcal{R}\left(x_{0}, y_{0}+1\right)\right.\right. \\
& \left.\left.-2 \log \mathcal{R}\left(x_{0}, y_{0}\right)\right]\right\}^{-1} .
\end{aligned}
$$

\subsection{Forms and sources of error}

There are three forms of errors in DPIV. They are the outliers, the mean-bias errors and root-mean-square (RMS) errors. Outliers are those velocity vectors which result from a mismatch in PIP. Therefore, the correlation peak of an outlier is small. It is inherent to their nature that the outliers usually appear randomly both in direction and in amplitude. Usually these errors are larger than one pixel and are therefore easy to detect. The major factors responsible for outliers are an insufficient number of particles being present in the PIP, strong velocity gradients and strong three-dimensional flow motions. Methods to reduce or remove the outliers have been discussed in many previous publications (Keane and Adrian 1990, Willert 1992, Huang et al 1993a, 1993b, Westerweel 1993) and will therefore not be discussed further in this paper. 


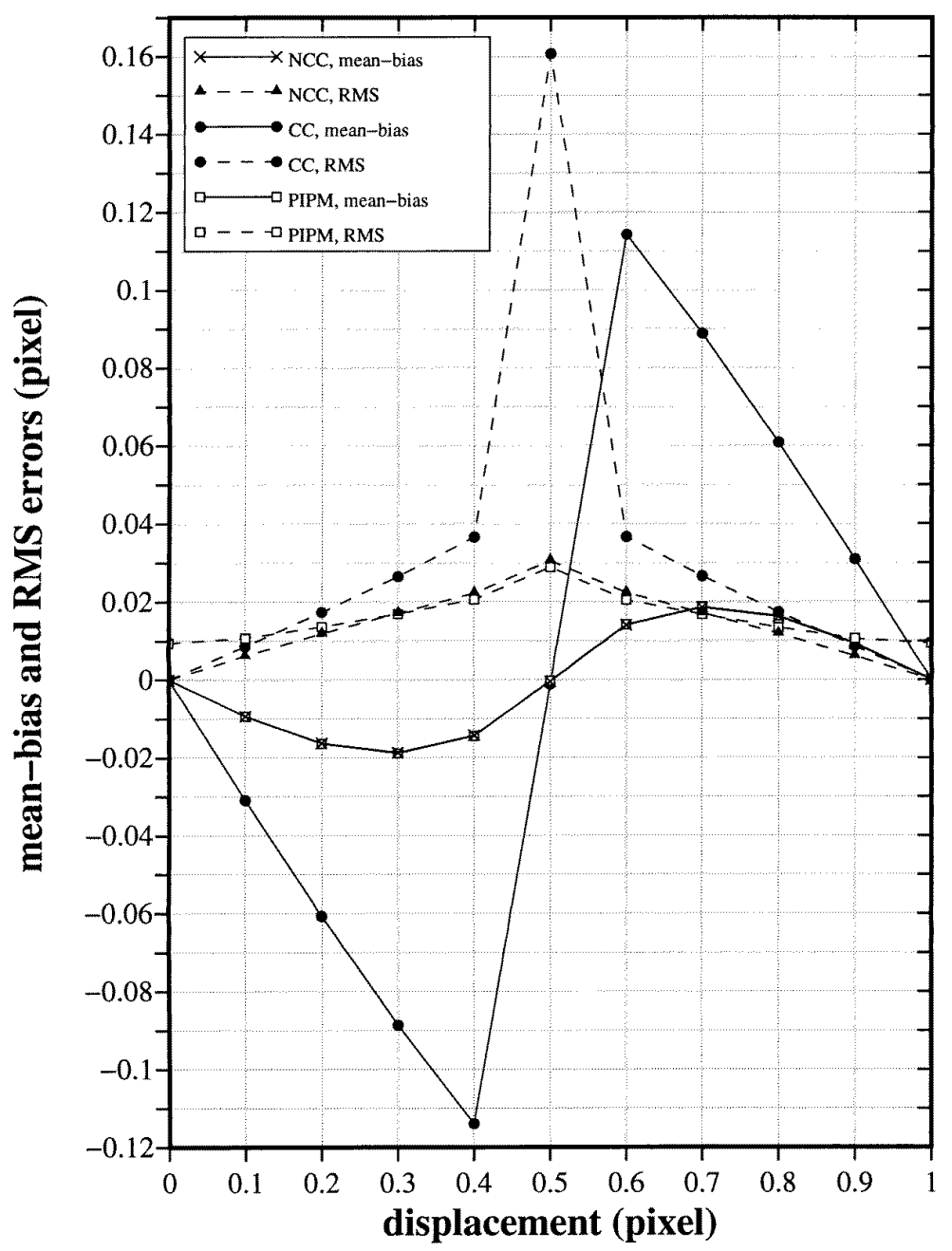

Figure 4. Illustration of cross correlation (CC): (a) $\mathcal{P} \mathcal{I} \mathcal{P}_{1}$, (b) $\mathcal{P} \mathcal{I} \mathcal{P}_{2}$, (c) $\mathcal{C}(0,0)$ is obtained when $\mathcal{P} \mathcal{I} \mathcal{P}_{2}$ fully overlaps $\mathcal{P} \mathcal{I} \mathcal{P}_{1}$ and (d) $\mathcal{C}(1,0)$ is obtained when $\mathcal{P I P} \mathcal{P}_{2}$ is one pixel to the right of $\mathcal{P} \mathcal{I} \mathcal{P}_{1}$.

Errors are also present in the velocity vectors obtained with sufficiently large correlation peaks. We decompose the error into two forms; namely, the mean-bias error and the RMS error. Given a unique displacement applied to all particles, the particle displacements obtained with DPIV at various interrogation locations can be different. Suppose that the actual particle displacement is $d_{a}$ and $N$ displacements $d_{i}(i=1,2, \ldots, N)$ have been evaluated. The difference between the actual displacement $d_{a}$ and the mean of these displacements,

$$
d_{m}=\frac{1}{N} \sum_{i=1}^{N} d_{i}
$$

is the mean-bias error $d_{b}$,

$$
d_{b}=d_{m}-d_{a} .
$$

The RMS error is defined as

$$
\sigma=\left(\frac{\sum_{i=1}^{N}\left(d_{i}-d_{m}\right)^{2}}{N}\right)^{1 / 2}
$$

which reflects the deviation of the particle displacements from their mean.
The existence of a non-zero mean-bias error $d_{b}$ indicates that the shape of the correlation peak does not agree with the fitted curve in the mean. The RMS error $\sigma$ may be affected by many factors. In addition to the improper particle seeding, the strong velocity gradients and the three-dimensional flow motion, other factors such as the camera's dark-current noise, the nonlinear and nonuniform response of the camera, the non-uniformity of the illumination, the non-uniform reflection of particles at different locations and angles, the introduction of noise from the cable (analogue video noise) and the digitization error can contribute to the RMS error. The nonlinear and non-uniform response of the camera usually has a minor effect and will not be studied. The non-uniform reflection of particles at different locations and angles is a complicated issue (Raffel et al 1995) which is beyond the scope of the present study. The use of laser-induced fluorescent sphere particles, when possible, minimizes this effect. The study of the three-dimensional effects on the RMS errors belongs to the topic of three-dimensional PIV (Raffel et al 1995, Meng and Hussain 1995, Prasad and Adrian 1993) and will not be discussed further in this paper. The influence of the velocity gradient on the accuracy of PIV has been 


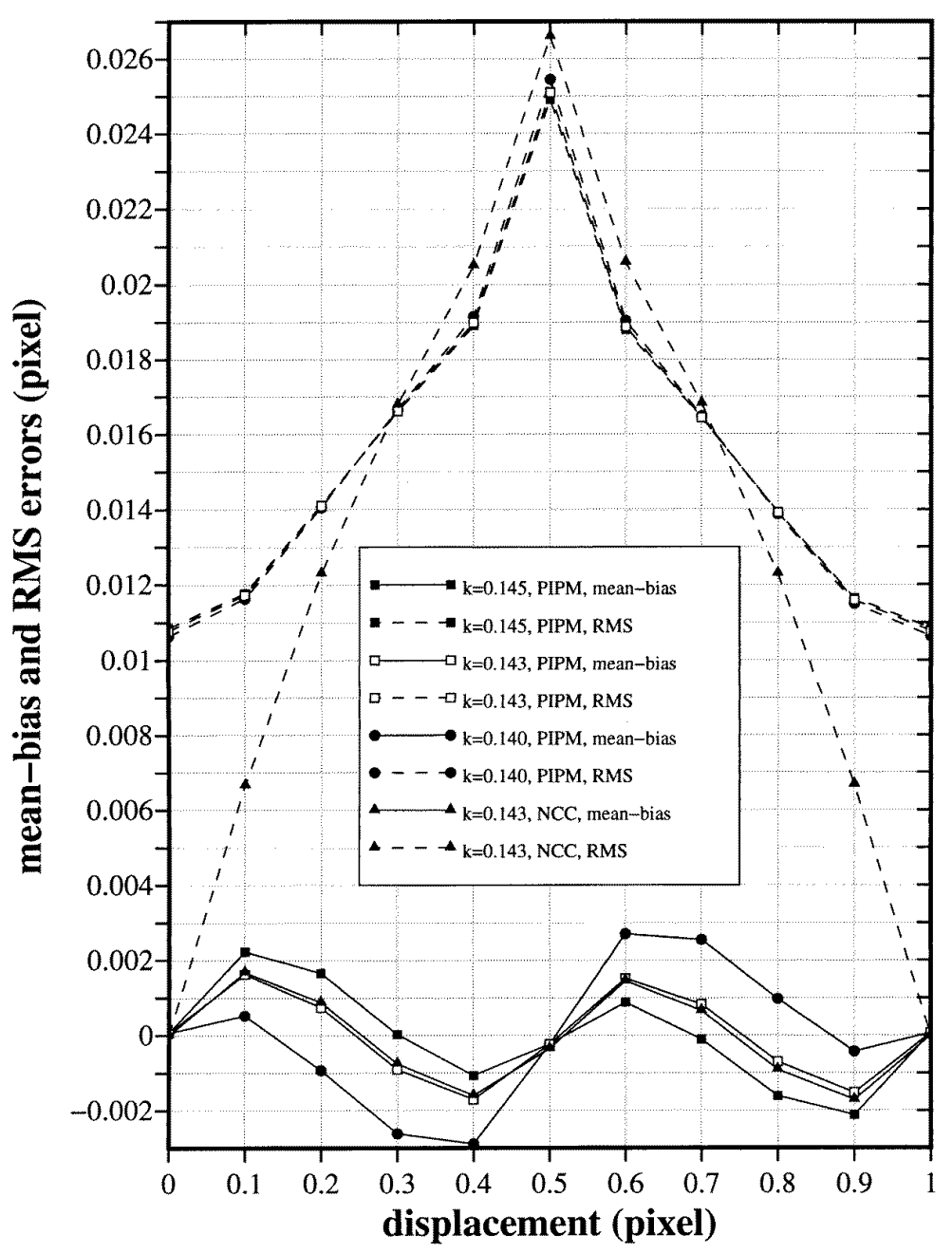

Figure 5. The mean-bias and RMS errors of the integer peak neighbour compensation method as functions of the particle displacement for PIPM, NCC and the compensation constant $(k=0.140,0.143$ and 0.145$)$.

detailed in the early publications (Huang et al 1993a, Keane and Adrian 1990). An efficient improvement of the accuracy of PIV relative to velocity gradients has been presented through particle image distortion (PID) (Huang et al 1993b).

All the aforementioned factors which cause the DPIV error have one feature in common - they produce noise in particle images, if we define image noise as all contributions other than the particle image itself. The uncertainty in the image noise is defined by the uncertainty in the pixel values. Since particles are described by pixel profiles, the uncertainty in the pixel values results in the uncertainty of the particle locations and hence results in errors of particle displacements.

The definition of the correlation function in a discrete domain and the method used to locate the sub-pixel correlation peak can also contribute both to mean-bias errors and to RMS errors. Not enough attention, however, has been paid to this point. Therefore, the major focus of this paper is to study the combined contribution of the DPIV processing method and the camera noise to the mean-bias and RMS errors.

\section{Errors in simulated particle images}

\subsection{The simulation of particle images}

Particle images can be obtained through numerical simulation, in which randomly distributed particles are generated onto a pixel array. The only noise of the simulated images is caused by the digitization of the pixel intensity. Suppose that the simulated particles have a Gaussian intensity profile,

$$
I(x, y)=I_{0} \exp \left(\frac{\left(x-x_{0}\right)^{2}+\left(y-y_{0}\right)^{2}}{\sigma_{p}^{2}}\right)
$$

where $\left(x_{0}, y_{0}\right)$ is the central location of the particles and the particles are randomly distributed over the whole image. The $\sigma_{p}$ is associated with the particle diameter $d_{p}$, where $\sigma_{p}=d_{p} / \sqrt{ } 2$. The corresponding particle pixel value is the integral of equation (7) over the area of the pixel:

$$
\begin{aligned}
& I(x, y) \\
& \quad=I_{0} \int_{x-0.5}^{x+0.5} \int_{y-0.5}^{y+0.5} \exp \left(\frac{\left(x-x_{0}\right)^{2}+\left(y-y_{0}\right)^{2}}{\sigma_{p}^{2}}\right) \mathrm{d} x \mathrm{~d} y .
\end{aligned}
$$




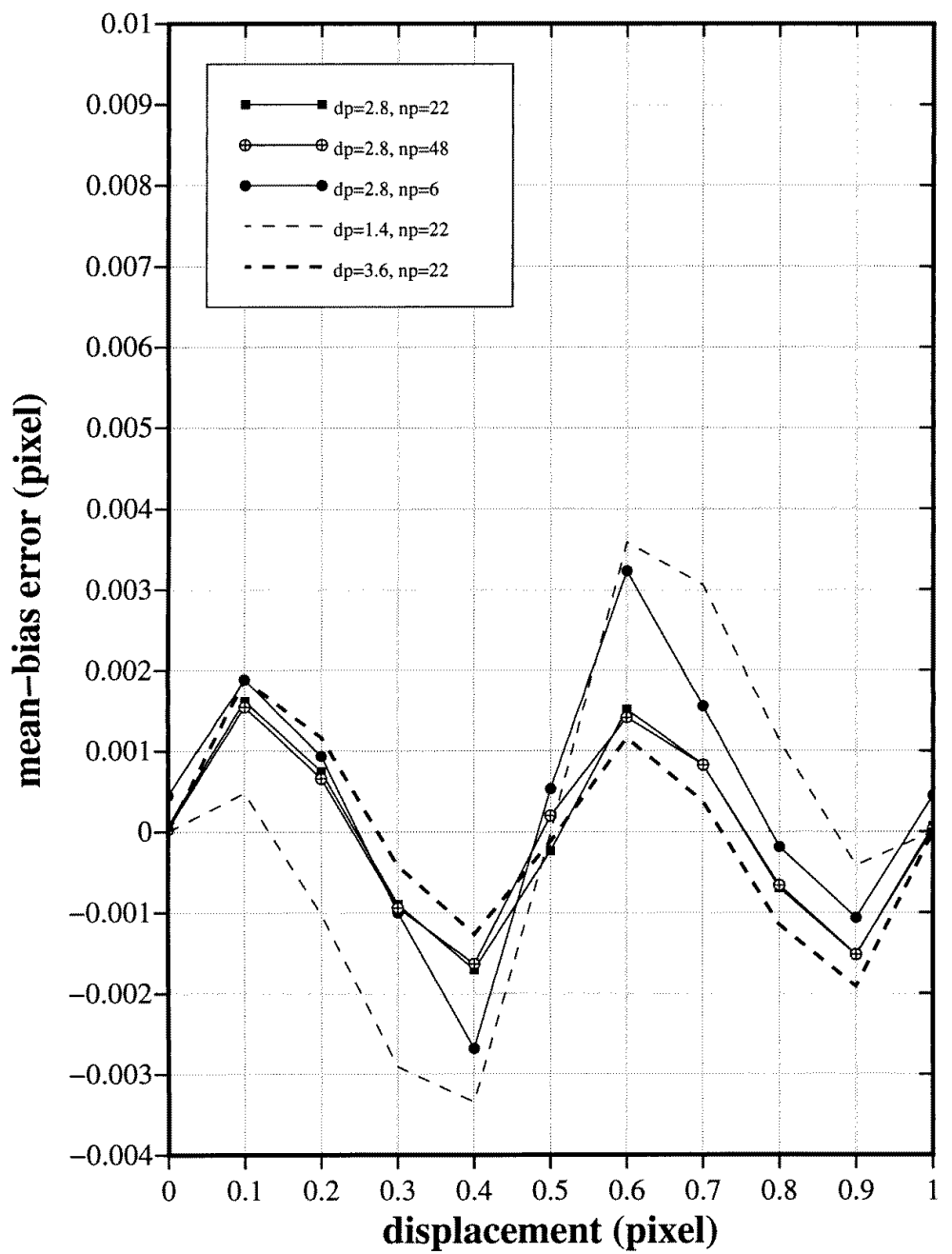

Figure 6. The mean-bias error of PIPM in response to different particle sizes $\left(d_{p}\right)$ and particle numbers $\left(n_{p}\right)$ in PIP for a compensation constant $k=0.143$.

Once a particle image $I_{1}(x, y)$ has been generated, its image pair $I_{2}(x, y)$ is shifted with a known unique distance $\left(x_{s}, y_{s}\right)$,

$$
I_{2}(x, y)=I_{1}\left(x+x_{s}, y+y_{s}\right) .
$$

Because PIPM does not produce any extra errors for particle displacements larger than one pixel and because errors generated from particle displacements larger than one pixel can be removed by using the image shifting technique (Westerweel et al 1997), the shift for the present study is confined to within one pixel; namely, $x_{s}, y_{s} \in[0,1]$.

\subsection{Mean-bias and RMS errors-a brief overview}

The image pairs with a unique particle displacement $d$ were processed by the DPIV in which the velocity grid spacing was $5 \times 5$ pixels. The image size was $768 \times 480$ pixels. In total, $146 \times 90$ velocities were evaluated for each image pair. The particle seeding density was 0.05 particle per pixel ${ }^{2}$ and the particle diameter $\left(d_{p}\right)$ was 2.8 pixels. Both the $\mathrm{CC}$ and PIPM method were examined. Figure 2 shows the displacement-frequency distribution of the DPIV response when the size of $\mathcal{P I P} \mathcal{P}_{1}$ is $21 \times 21$ pixels. (Therefore, about 22 particles were present in the interrogation area.) When the actual displacement was $d=0.3$ pixel, PIPM gave a mean-bias error $d_{b}=-0.019$ pixels (the mean displacement was found to be 0.281 pixels from figure 2) and a RMS $\sigma=0.017$ pixels, whereas $\mathrm{CC}$ gives a worse result: the mean-bias error was $d_{b}=-0.089$ pixels and the RMS error was $\sigma=0.027$ pixels. The largest RMS error occurred when $d=0.5$ pixels. As a reminder, the meanbias and RMS errors have been defined by equations (5) and (6), respectively.

\subsection{The correction of the mean-bias error}

Investigations have shown that the evaluated correlation peak location is always biased to the nearest integer value. This peak-bias effect results in a mean-bias error of

$$
\begin{array}{ll}
m_{b}<0 & d<0.5 \\
m_{b}>0 & d>0.5
\end{array}
$$

as shown in figure 3. Even though figure 3 shows that, for $d=0.5$, the mean bias is zero, figure 2 shows 


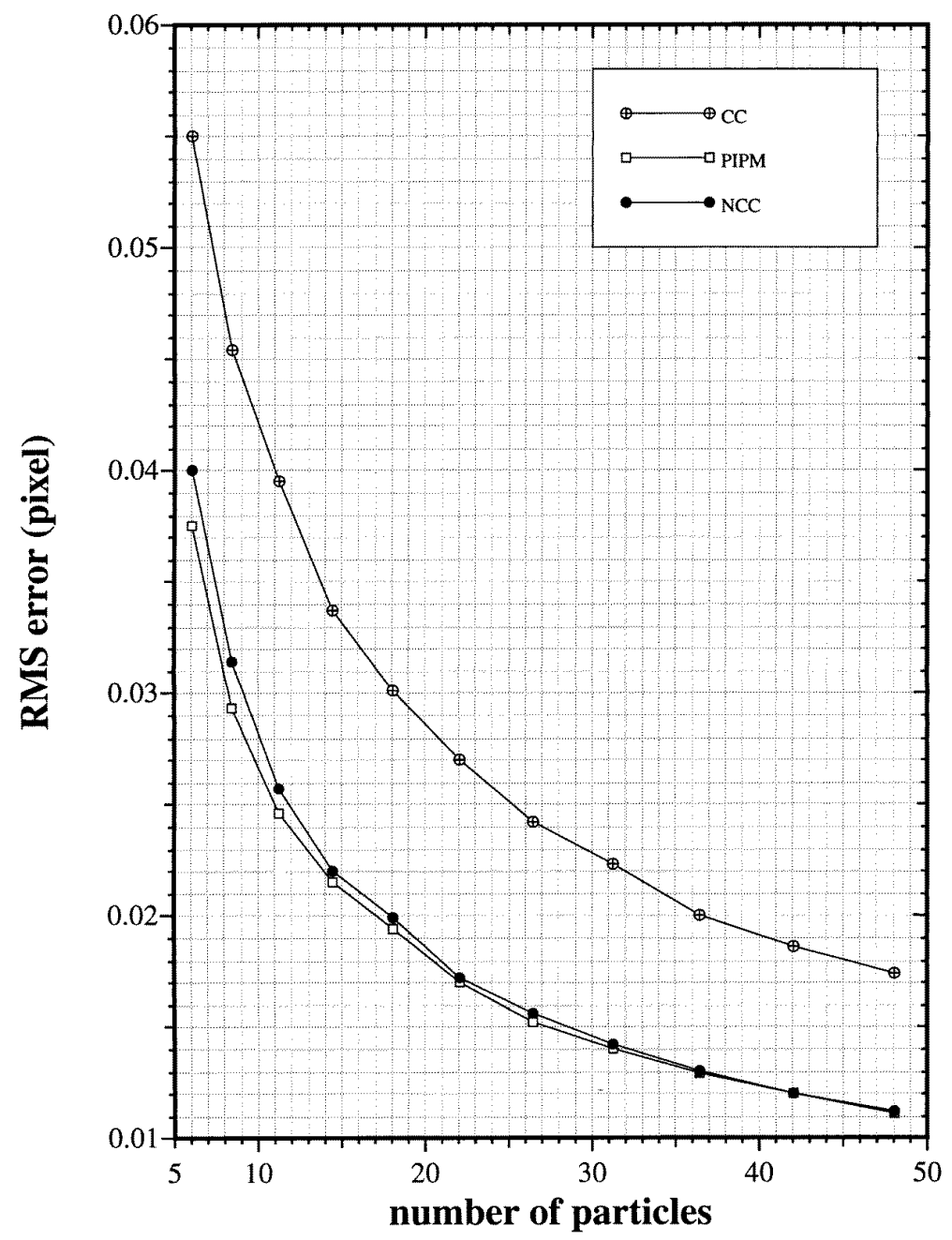

Figure 7. The RMS error of DPIV as a function of the particle number $\left(n_{p}\right)$ present in PIP when the actual particle displacement is 0.3 pixels.

that the most frequent displacements were to both sides of 0.5 pixels. Although the peak-bias phenomenon was noticed by Westerweel et al (1997), no consequent solution has been reported. In this section we will introduce some methods to correct the mean-bias error.

3.3.1. The normalized cross correlation. Insights into the peak-bias phenomenon can be explained using figure 4 . Suppose that there are two PIPs, $\mathcal{P} \mathcal{I} \mathcal{P}_{1}$ and $\mathcal{P} \mathcal{I} \mathcal{P}_{2}$, as shown in figures 4(a) and (b), such that the actual particle displacement between this two images is 0.5 pixels. We expect the same values of $\mathcal{C}(0,0)$ and $\mathcal{C}(1,0)$ as those which are defined in equation (1) because $\mathcal{C}(0,0)$ and $\mathcal{C}(1,0)$ are symmetrical with respect to the actual correlation peak location. On the other hand, the values of $\mathcal{C}(0,0)$ and $\mathcal{C}(1,0)$ are proportional to the overlapping areas of $\mathcal{P} \mathcal{I} \mathcal{P}_{1}$ and $\mathcal{P} \mathcal{I P}_{2}$ (Huang et al 1993a), as shown in figures 4(c) and $4(\mathrm{~d})$. However, $\mathcal{C}(0,0)$ is obtained when $\mathcal{P} \mathcal{I} \mathcal{P}_{1}$ and $\mathcal{P} \mathcal{I P}_{2}$ are fully overlapped whereas $\mathcal{C}(1,0)$ is not. Thus, according to equation (4) we obtain a correlation peak location biasing to $\mathcal{C}(0,0)$.
The PIPM usually has less mean-bias error than does that of $\mathrm{CC}$, as shown in figures 2 and 3 . This occurs because the correlation function of PIPM is normalized and the correlation function of $\mathrm{CC}$ is not. To improve the performance of $\mathrm{CC}$ without losing the advantage of implementing FFTs for fast processing, we only need to normalize the correlation values used to evaluate the subpixel location of the correlation peak. Therefore, first, the integer peak is found using CC. Then this point and its four neighbouring points are normalized. These five normalized correlation points are then used to evaluate the correlation peak at a sub-pixel location. Similarly to equation (4) we have

$$
\begin{aligned}
x= & x_{0}+\left\{\log \mathcal{C}^{\prime}\left(x_{0}-1, y_{0}\right)-\log \mathcal{C}^{\prime}\left(x_{0}+1, y_{0}\right)\right\} \\
& \times\left\{2 \left[\log \mathcal{C}^{\prime}\left(x_{0}-1, y_{0}\right)+\log \mathcal{C}^{\prime}\left(x_{0}+1, y_{0}\right)\right.\right. \\
& \left.\left.-2 \log \mathcal{C}^{\prime}\left(x_{0}, y_{0}\right)\right]\right\}^{-1} \\
y= & y_{0}+\left\{\log \mathcal{C}^{\prime}\left(x_{0}, y_{0}-1\right)-\log \mathcal{C}^{\prime}\left(x_{0}, y_{0}+1\right)\right\} \\
& \times\left\{2 \left[\log \mathcal{C}^{\prime}\left(x_{0}, y_{0}-1\right)+\log \mathcal{C}^{\prime}\left(x_{0}, y_{0}+1\right)\right.\right. \\
& \left.\left.-2 \log \mathcal{C}^{\prime}\left(x_{0}, y_{0}\right)\right]\right\}^{-1}
\end{aligned}
$$




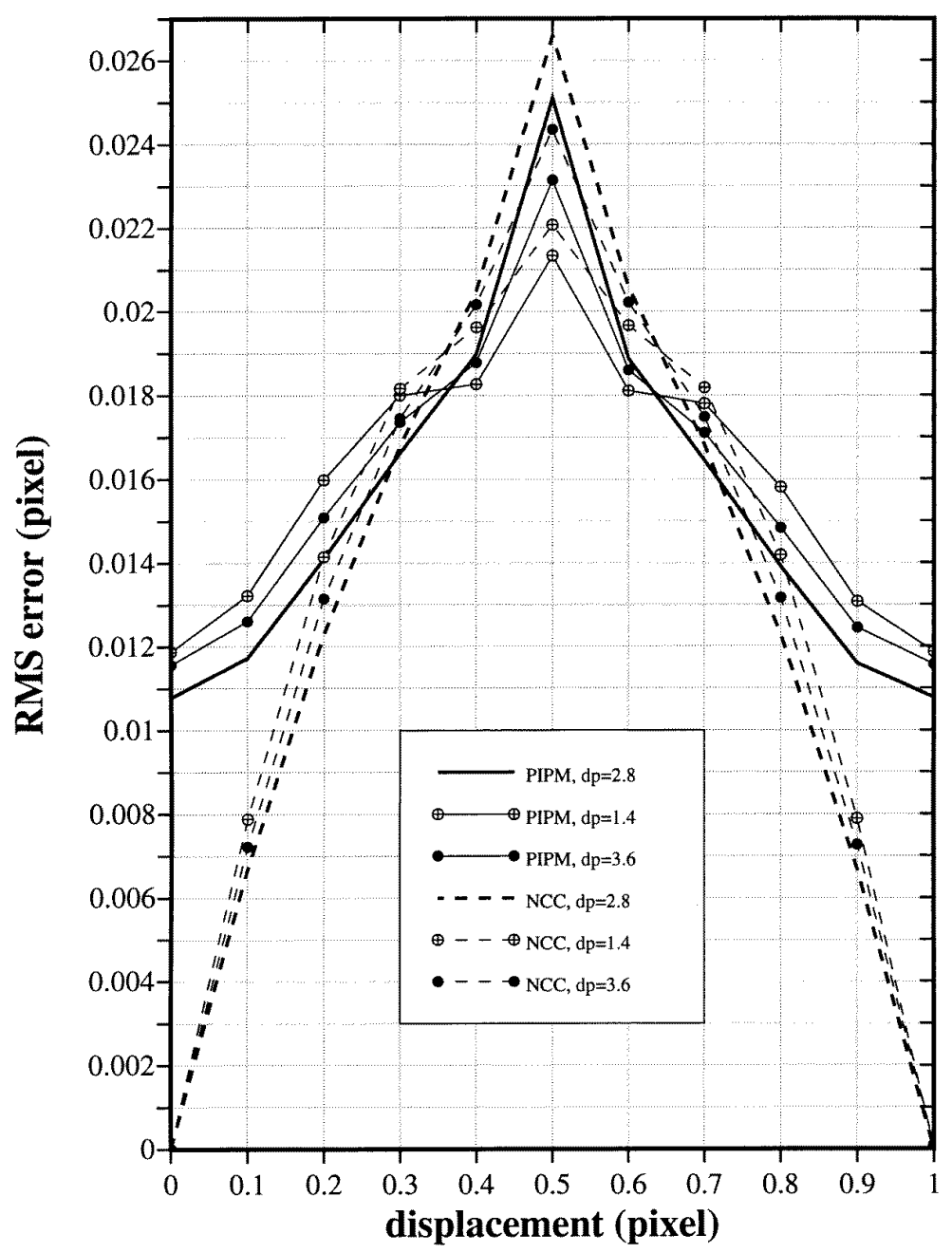

Figure 8. The RMS error of DPIV as a function of the particle diameter $\left(d_{p}\right)$.

where $\mathcal{C}^{\prime}$ indicates a normalized correlation value from $\mathrm{CC}$,

$$
\mathcal{C}^{\prime}(m, n)=\frac{\mathcal{C}(m, n)}{\left[\sum_{i, j \in A} \mathcal{P I P}_{1}^{2}(i, j) \sum_{i, j \in A} \mathcal{P I P}_{2}^{2}(i, j)\right]^{1 / 2}}
$$

where $A$ is the overlapping area of $\mathcal{P} \mathcal{I} \mathcal{P}_{1}$ and $\mathcal{P} \mathcal{I} \mathcal{P}_{2}$ for the corresponding $\mathcal{C}$. We call this procedure the normalized cross correlation (NCC). Figure 3 shows that NCC significantly improves the CC performance. While having almost the same mean-bias error as that of PIPM, NCC has a smaller RMS error than that of PIPM when a displacement is closest to an integer $(d<0.3$ or $d>$ $0.7)$.

Again, it is important to note that the normalization in equation (9) is performed on only five values after $\mathrm{CC}$ has been completed and therefore takes very limited computation time. Therefore, it is imperative that a uniform light sheet is available and that the two sequential particle image pairs be exposed at equal intensities. Otherwise, CC may give mean-bias errors larger than one pixel and the resulting NCC results given in equation (9) will then be meaningless. In this case, PIPM would provide a better result (Gonzalez and Wintz 1987), since PIPM normalizes the cross correlation values throughout the interrogation area and therefore yields more accurate integer peaks.

3.3.2. The compensation for integer peak neighbours. The normalized correlation function both in PIPM and in NCC still gives a mean-bias error of the order of 0.01 pixels (see figure 3). This error is comparable to the RMS error and cannot be neglected. As explained in section 2.3, the mean-bias error exists since the fitted curve does not agree with the actual correlation shape about its peak. This is especially apparent when particle displacements are not integer values (when the discrete correlation shape about the peak is asymmetrical). In this paper, we would like to introduce a method to eliminate the mean-bias error by properly compensating for the asymmetry around the integer peak neighbours while using a symmetrical Gaussian-peak function. Suppose $\mathcal{R}\left(x_{0}, y_{0}\right)$ is the integer peak and $\mathcal{R}_{n}$ are its neighbours, namely

$$
\begin{aligned}
\mathcal{R}_{n} \in & \left\{\mathcal{R}\left(x_{0}+1, y_{0}\right), \mathcal{R}\left(x_{0}-1, y_{0}\right), \mathcal{R}\left(x_{0}, y_{0}+1\right),\right. \\
& \left.\mathcal{R}\left(x_{0}, y_{0}-1\right)\right\}
\end{aligned}
$$




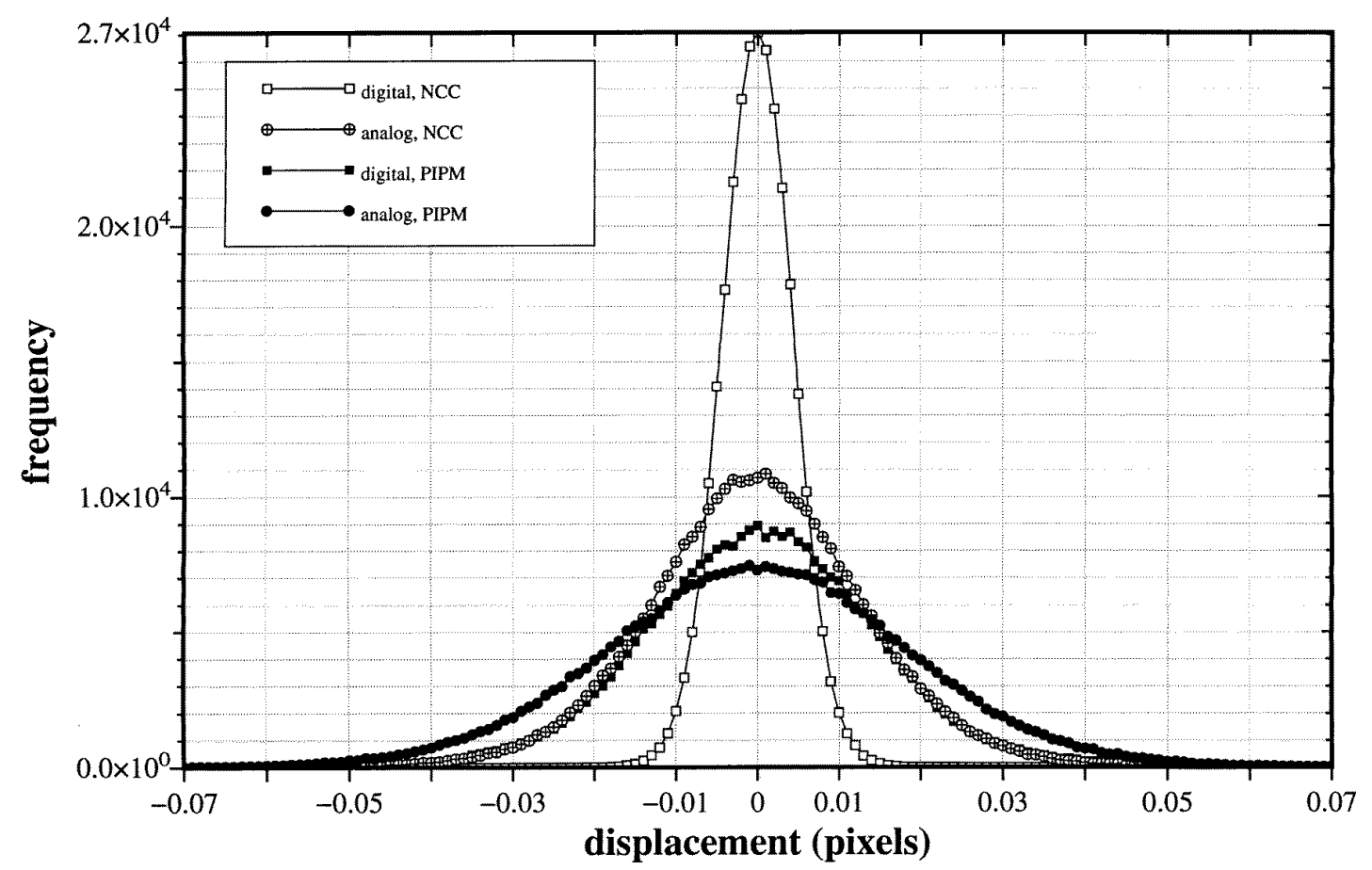

Figure 9. A comparison between analogue and digital videos showing the DPIV error distribution in response to a zero particle displacement. Both PIPM and NCC methods are applied.

the compensated integer peak neighbour is given by

$$
\mathcal{R}_{n}^{\prime}=\alpha \mathcal{R}_{n} \quad \alpha>1
$$

We found that a constant $\alpha$ in equation (11) gave a limited improvement to the mean-bias error. An efficient way is to define $\alpha$ as

$$
\alpha=1+k \frac{\mathcal{R}\left(x_{0}, y_{0}\right)-\mathcal{R}_{\text {nmax }}}{\mathcal{R}\left(x_{0}, y_{0}\right)}
$$

where $k(0<k<1)$ is a compensation constant and $\mathcal{R}_{\text {nmax }}$ is the maximum value among the integer peak neighbours $\left\{\mathcal{R}_{n}\right\}$.

Figure 5 shows the PIPM and NCC results with the integer peak neighbour compensation. When $k=0.143$, which is the optimal compensation constant in this case, the mean-bias error is of the order of 0.001 pixels - an order of magnitude less than the one without the compensation (see figure 3 and note the difference in scale between figures 3 and 5). The compensation has no influence on the RMS error. Increasing $k$ causes over-compensation whereas reducing $k$ results in under-compensation. Under the same conditions, both PIPM and NCC methods have the same order of the mean-bias error. Figure 5 shows more clearly that the NCC method has less RMS error when the displacement is close to an integer $(d<0.3$ or $d>0.7)$ whereas the PIPM method has less RMS error when the displacement is in the vicinity of 0.5 .

In an application of DPIV, the particle size and the particle seeding density may vary. What is the optimal compensation constant $k_{\text {opt }}$ under different conditions? A good compensation system should have a constant $k_{\text {opt }}$ under different conditions. With such a question in mind, we have studied the DPIV's mean-bias error in response to different particle sizes $\left(d_{p}\right)$ and different particle numbers $\left(n_{p}\right)$ for a given compensation constant, where $n_{p}$ was determined by the particle seeding density and the size of the PIP. Only the result of the PIPM method is plotted in figure 6 since the NCC method's behaviour was almost the same. We found $k_{\text {opt }}=0.143$ for $d_{p}=2.8$ and $n_{p}=22$. The mean-bias error was almost independent of $n_{p}$ when it was sufficiently large, namely, $n_{p}>20$. When $n_{p}$ was small, namely, $n_{p}<10$, reducing $n_{p}$ resulted in an undercompensation. When $n_{p}=6$ the mean-bias error could be twice as much as when $n_{p} \geq 22$. Small particle sizes caused an under-compensation. Large particle sizes, on the other hand, caused an over-compensation. The overor under-compensation could be balanced by decreasing or increasing the compensation constant. It should be pointed out that the change of particle size from $d_{p}=2.8$ to $d_{p}=3.6$ and the change of particle number in PIP from $n_{p}=6$ to $n_{p}=48$ resulted in a change in the mean-bias error of the order of 0.001 pixels which was negligible in comparison with the RMS error (see figure 5). This fact tells us that the optimal compensation given by equations (11) and (12) is insensitive to the changes in $d_{p}$ and $n_{p}$.

\subsection{The RMS error}

With a proper correction of the mean-bias error, the RMS error becomes the only major error in DPIV. In this section we will detail and summarize the RMS error of DPIV when simulated particle images are used.

Figure 7 shows that the number $\left(n_{p}\right)$ of particles present in PIP plays an important role in the RMS error of DPIV. 


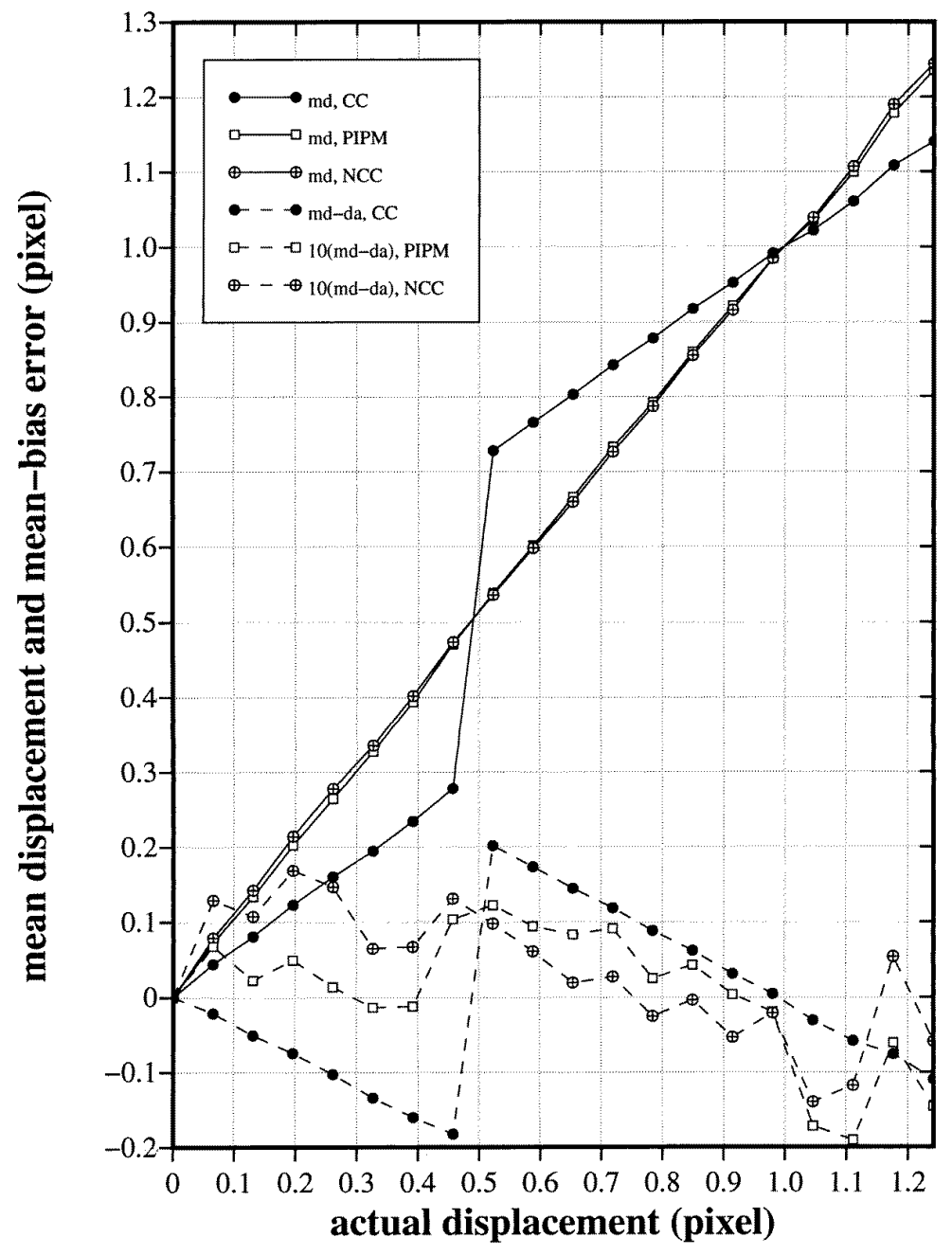

Figure 10. The DPIV mean displacement, md, and the mean-bias error, md - $d_{a}$, where $d_{a}$ is the actual displacement, in response to the uniform translation of the particle pattern.

The larger the $n_{p}$ the less the RMS error. There are two ways to increase $n_{p}$, either to increase the PIP size or to increase the seeding density. For the present case the particle diameter is $d_{p}=2.8$ pixels and the actual particle displacement is $d_{a}=0.3$ pixels. The particle diameter $d_{p}$ has a minor influence on the RMS error, as shown in figure 8 . This result does not agree with the conclusion that the RMS error is proportional to the particle diameter (Adrian 1991). Our results could have been due to the normalization of the correlation function and the compensation for integer peak neighbours. The insensitivity of the RMS error to the particle diameter is important since it makes the selection of the particle size not crucial to the DPIV error. Figure 8 also shows that the farther the particle displacement from an integer the larger the RMS error. The largest RMS error occurs when $d_{a}=0.5$ pixels, at a value about twice as large as that when $d_{a}=0.3$ pixels. The normalized sub-pixel peak locating (NCC) also significantly reduces the RMS error of CC. When $d_{a}=0.3$ pixels, both PIPM and NCC have almost the same RMS error. The RMS error of PIPM is greater than that of NCC when $d_{a}<0.3$ pixels, whereas the RMS error of PIPM is less than that of NCC when $d_{a}>0.3$ pixels (also see figures 3 and 5). When the particle displacement is an integer, the RMS error of the NCC method is zero. With PIPM or NCC, an estimate of the RMS error of DPIV on the simulated images would be 0.03 pixels at $d_{a}=0.5$ pixels. This estimate was obtained on the basis of a typical combination of particle density and PIP size, $n_{p} \approx 20$. The RMS error was not affected by the compensation for integer peak neighbours.

\section{Errors in particle images taken with a video camera}

In this section we will study the DPIV errors arising from the imaging device, a CCD video camera. For this purpose, randomly distributed particle patterns were generated through the numerical simulation as described in section 3.1. The resulting particle pattern was printed on a piece of paper which was mounted on a stable $x-y$ translation stage. While it was being illuminated with a uniform light source with a DC power supply, the particle pattern was imaged with a CCD video camera which was 


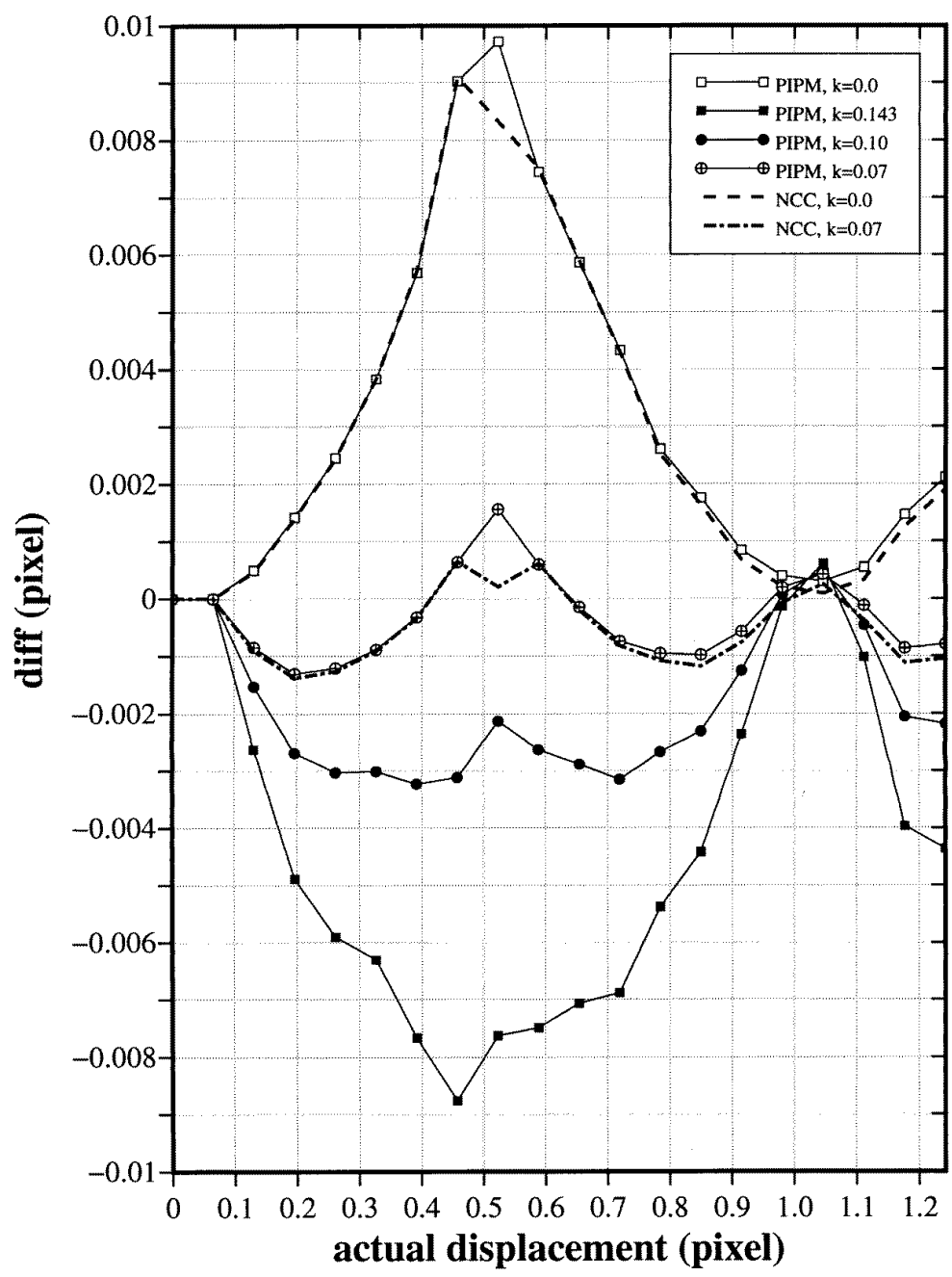

Figure 11. The DPIV mean-bias error diff, defined in equation (13), as a function of the actual displacement for various compensation constants $k$.

positioned perpendicular to the image plane. Owing to the CCD's dark-current noise, the amplifier noise, the noise introduced while the video signal was being transmitted and digitization errors, the output image from the camera became noisy. With a known shift of the particles, the errors of DPIV due to the video camera noise can be determined.

\subsection{The DPIV error from digital and analogue video}

Electrical charges in $\mathrm{CCD}$ cells correspond to the pixel values of the image. There are two ways to convert the electrical charge signal to an image. The first way is to convert the electrical charge signal to a voltage signal and then encode it to a standard analogue video signal. With an analogue video cable the analogue video signal is transmitted to a frame grabber which digitizes the video signal into an image. The transmission of the video signal in the analogue format is popular and economical because only one cable is required. Noise, however, was introduced while the analogue video signal was being transmitted. The second way is to convert the electrical charge signal to a voltage signal and then digitize it directly into a digital video signal inside the camera. The direct digitization minimizes the noise generation that is inherent to the first method. With a digital video cable the digital video signal is transmitted to a frame grabber which acquires the digital video signal and saves it into an image. In this way, no noise is introduced during transmission of the video signal.

To verify this, images of the particle pattern were taken with a Pulnix CCD video camera which had both analogue and digital video output. With an EPIX frame grabber, both the analogue and the digital video signal were acquired. No displacement was applied to the particle pattern. For each case (analogue and digital video) 22 image pairs were recorded. For each image pair, DPIV was implemented with a $5 \times 5$ pixel $^{2}$ data grid spacing. The interrogation area size was $21 \times 21$ pixel $^{2}$. The image size was $768 \times$ 480 pixel $^{2}$. A total of about 300000 displacements were evaluated for each case. Figure 9 shows the displacement distributions. With NCC, the RMS error was 0.0044 pixels for the digital video and 0.0135 pixels for the analogue video. With PIPM, the RMS error was 0.0143 pixels for the digital video and 0.0189 pixels for the analogue video. 


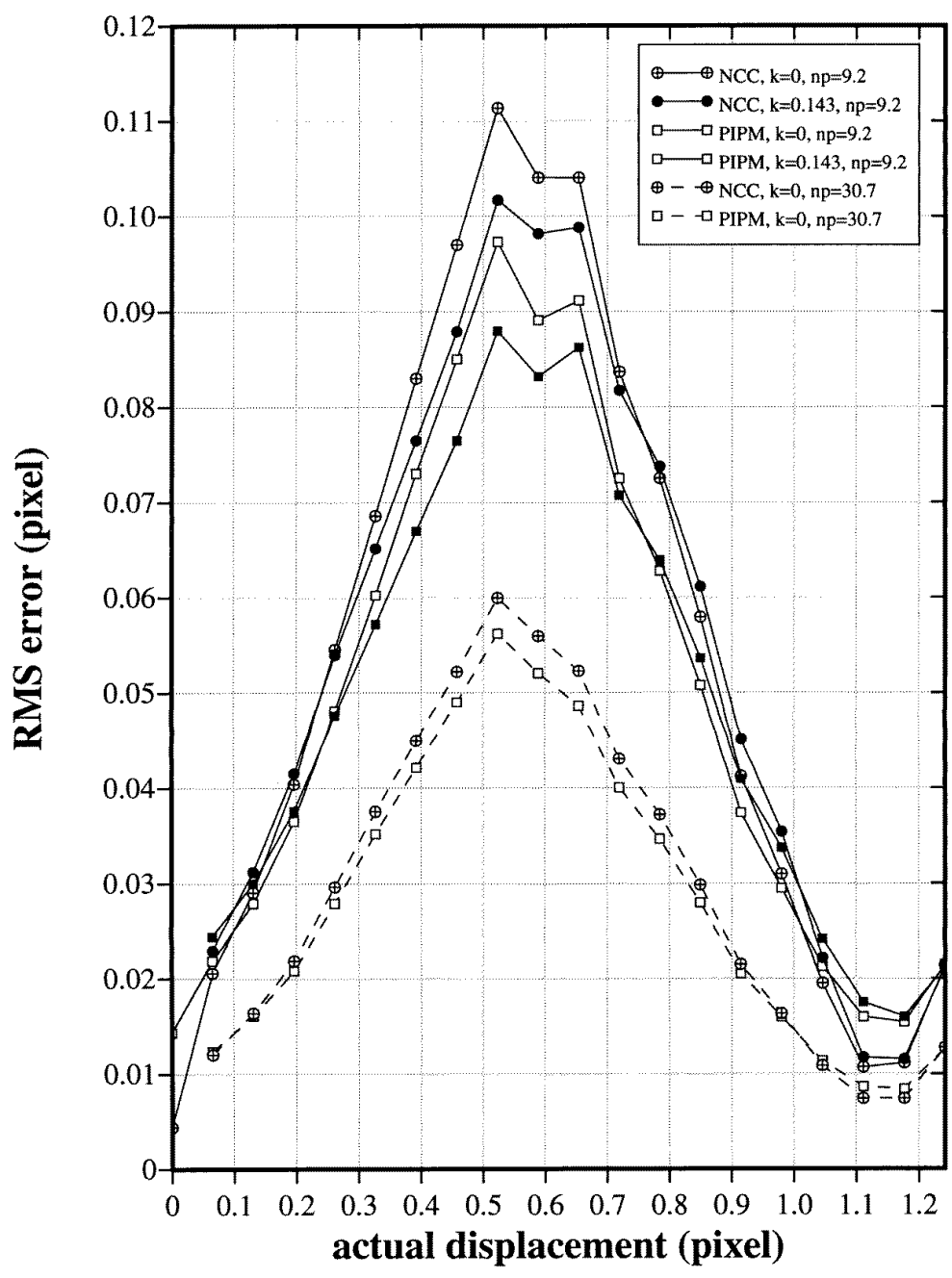

Figure 12. The DPIV RMS error for noisy images as a function of the actual displacement under various conditions.

This clearly demonstrates that, with the same processing method, the analogue video signal results in a larger DPIV error than the digital video does. The RMS error of NCC is smaller than that of PIPM because the mean displacement is zero. In this case, NCC is symmetrical whereas PIPM is not, according to equations (1), (10) and (3). Thus, in NCC, only the noise of the image contributes to the RMS error, whereas in PIPM, the non-uniformity of the images also makes error contributions. Actually, as can be seen in figure 8 , the simulation result of synthetic images already indicates that NCC has a smaller RMS error than PIPM does when the displacement is close to an integer number. In the following section, only the results from the digital video signal will be discussed.

\subsection{The mean-bias and RMS errors}

The mean-bias and RMS errors are directly associated with the particle displacement. In the study described in this section, the displacement of the particle pattern was achieved by shifting a randomly generated particle image (as described in the previous section) with an $x-y$ translation stage. The resolution of the translation stage was $1 \mu \mathrm{m}$, corresponding to 0.00658 pixels in the image domain. The image was shifted 20 times such that each increment was $10 \mu \mathrm{m}$, namely, 0.0658 pixels. At each displacement position $i, i=0,1,2, \ldots, 19$, 44 images were acquired. Images were then paired between displacement 0 and $i$, constructing a group of image pairs. Thus, there are 44 image pairs in each group. In total, 13 million displacement evaluations were used to build up the statistics in each group. In figure 10 the DPIV mean displacement and mean-bias error are plotted for each particle displacement index $i$. The DPIV mean displacement was $\operatorname{md}(i)$ and the mean-bias error was $\operatorname{mb}(i)=\operatorname{md}(i)-0.0568 i$. This shows that $\mathrm{CC}$ (with image shifting) gives a maximum mean-bias error of about 0.2 pixels, whereas the mean-bias of error PIPM and NCC is an order of magnitude smaller. Note that the scale of the mean-bias error of PIPM and NCC is ten times smaller than that of CC.

Actually, the particle displacements at the corresponding index $i$ are not exactly equal to $0.0658 \mathrm{i}$ pixels because the $x-y$ translation stage was driven manually and there 


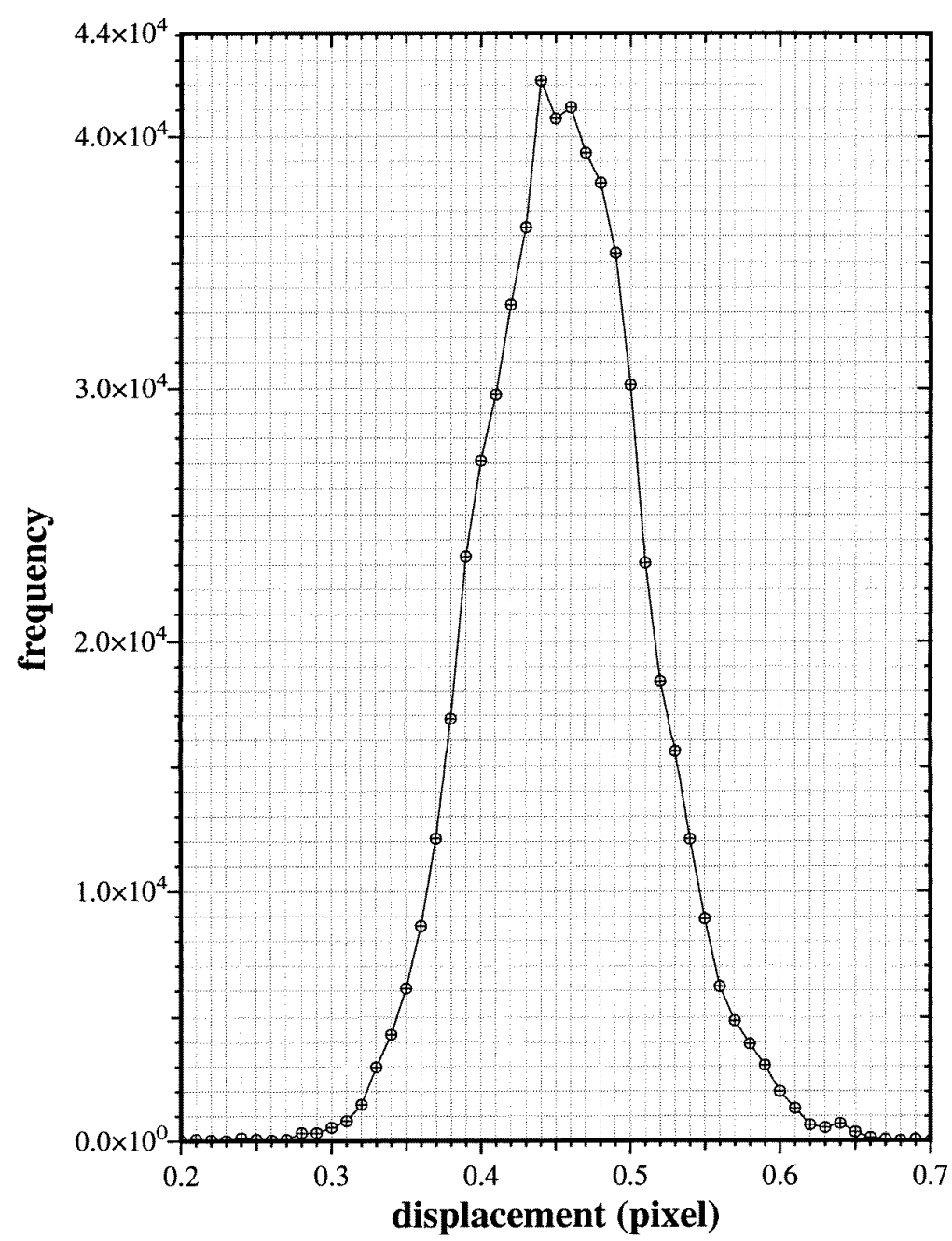

Figure 13. A frequency histogram of measured displacements of particle images taken with the digital video camera, where the actual displacement $d_{a}=0.46$ pixels and the RMS error is equal to 0.056 pixels.

was a $10 \%$ read-out error. In order to study the mean-bias error of PIPM and NCC more precisely, images were paired between displacement indices $i-1$ and $i$ and then evaluated with DPIV. Supposing that their DPIV mean displacement was $\operatorname{md}_{1}(i)$, the difference between $\operatorname{md}(i)-\operatorname{md}(i-1)$ and $\operatorname{md}_{1}(i)$

$\operatorname{diff}(i)=\operatorname{md}(i)-\operatorname{md}(i-1)-\operatorname{md}_{1}(i) \quad i=1,2, \ldots, 19$

reflects the mean-bias error. Without the mean-bias error the difference $\operatorname{diff}(i)$ would be a constant of zero for each $i$. Figure 11 shows the $\operatorname{diff}(i)$ of PIPM and NCC for various integer peak neighbour compensation constants $k$ (see section 3.3.2). When $k=0$, that is, without the compensation, the maximum value of $\operatorname{diff}(i)$ is about 0.01 pixels both for PIPM and for NCC. When $k=$ 0.07, which is about the optimal compensation value, the maximum amplitude of $\operatorname{diff}(i)$ drops to the order of 0.001 pixels both for PIPM and for NCC, which is an order of magnitude smaller than the value without compensation. The optimal compensation constant in this case (noisy images) is about half of the optimal compensation $(k=0.143)$ when the images are noisefree ones. A compensation value of $k=0.143$ results in an over-compensation in the case of noisy images, as demonstrated in figure 11. NCC behaves in almost the same way as PIPM does for $k=0.143$ and $k=0.1$ and is not shown in figure 11. This fact shows that proper noise in an image would lead to a reduction in the mean-bias error and would agree with the results of Westerweel (1993).

Noise in an image, on the other hand, significantly increases the RMS error of DPIV. When the number of particles present in PIP is $n_{p}=9.2$, the maximum RMS error is about 0.1 pixels both for PIPM and for NCC, as shown in figure 12. The RMS error of $\mathrm{CC}$ is more than twice as large and has not been shown in figure 12. A large number of particles in PIP reduces the RMS error, whereas the peak compensation has a minor effect on the RMS error. This tendency is identical to that in the case of simulated images. When the number of particles in PIP is increased to $n_{p}=30.7$, the RMS error is halved. In comparison with the case of simulated images, the RMS error in this case is about twice as large. In order to give a better insight into the RMS errors, figure 13 shows a frequency histogram 
of measured displacements when the actual displacement $d_{a}=0.46$ pixels, for example. The RMS error is equal to 0.056 pixels in this case.

\section{Conclusion}

The mean-bias and RMS errors are two major forms of error in DPIV. Their values are influenced by many factors. Among them the method of implementing the discrete cross correlation function and the way of locating the correlation peak to within a sub-pixel accuracy are fundamental in determining the upper limit of the DPIV accuracy. The CC is a common method in many DPIV systems because it can take advantage of the FFT algorithm and can therefore be implemented at a high speed. The present study shows that the mean-bias and RMS errors of $\mathrm{CC}$ in the simulated images when only digitization noise is present are of the order of 0.1 pixels. These errors are about an order of magnitude smaller in the PIPM, which is usually implemented directly due to the normalization of the correlation function. The normalization of CC improves the accuracy without adding much computation effort. The NCC has the same error level as that of the PIPM. The integer peak neighbour compensation method significantly suppresses the mean-bias error of the PIPM and NCC. With a proper compensation, the mean-bias error is negligible compared with the RMS error. An optimal compensation constant is $k_{\text {opt }}=0.143$ for the simulated images. It is insensitive to changes in the particle diameter and the number of particles present in the PIP. The RMS error in the present study does not exhibit a dependence on the particle diameter. It is a function of the particle number $\left(n_{p}\right)$ present in the PIP. Large $n_{p}$ reduces the RMS error. The maximum RMS error of the PIPM and NCC for a typical $n_{p}=20$ is about 0.03 pixels with the simulated images. Use of the $\mathrm{CC}$, however, produces larger mean-bias and RMS errors and is not recommended in DPIV.

Images taken with a CCD video camera are different from simulated images in that they are noisy. Noise in an image suppresses the mean-bias error (that is, a smaller compensation constant is required), but significantly amplifies the RMS error. With the digital video of a Pulnix camera (P9701) the optimal compensation constant drops to $k_{o p t}=0.07$ and the RMS error increases to the order of 0.1 pixels in the PIPM and NCC (it depends on $n_{p}$, of course). Since the mean-bias error is negligible compared with the RMS error with a proper mean-bias error correction, the lower the noise level in an image the smaller the DPIV error. Use of an analogue video is usually accompanied by stronger noise and therefore causes a larger RMS error than that of a digital video.

\section{Acknowledgment}

This work was sponsored by an URI grant from the Office of Naval Research, USA (grant number N00014-92-J1618).

\section{References}

Adrian R 1991 Particle-imaging techniques for experimental fluids mechanics Ann. Rev. Fluid Mech. 23 261-304

Boillot A and Prasad A 1996 Optimization procedure for pulse separation in cross-correlation PIV Exp. Fluids 21 87-93

Gonzalez R and Wintz P (eds) 1987 Digital Image Processing (New York: Addison Wesley) pp 90-2, 100-9, 425-7

Grant I (ed) 1994 Selected Papers on Particle Image Velocimetry (Washington: SPIE)

_ 1997 Particle image velocimetry - a review Proc. Inst. Mech. Eng. C 211 55-76

Guezennec Y and Kiritsis N 1990 Statistical investigation of errors in particle image velocimetry Exp. Fluids $\mathbf{1 0}$ $138-46$

Huang H, Fiedler H and Wang J 1993a Limitation and improvement of PIV, part I: limitation of PIV due to velocity gradients Exp. Fluids $\mathbf{1 5} 168-74$

_ 1993b Limitation and improvement of PIV, part II: particle image distortion - a novel technique Exp. Fluids $\mathbf{1 5}$ 263-73

Keane R and Adrian R 1990 Optimization of particle image velocimeters, part I, double pulsed systems Meas. Sci. Technol. 1 1202-15

Lourenco L and Krothapalli A 1995 On the accuracy of velocity and vorticity measurements with PIV Exp. Fluids $\mathbf{1 3}$ $421-8$

Meng H and Hussain F 1995 In-line recording and off-axis viewing technique for holographic particle velocimetry Appl. Opt. 34 1827-40

Prasad A and Adrian R 1993 Stereoscopic particle image velocimetry applied to liquid flows Exp. Fluids 15 49-60

Prasad A, Adrian R, Landreth C and Offutt P 1992 Effect of resolution on the speed and accuracy of particle image velocimetry interrogation Exp. Fluids 13 105-16

Raffel M, Gharib M, Ronneberger O and Kompenhans J 1995 Feasibility study of three-dimensional PIV by correlating images of particles within parallel light sheet planes Exp. Fluids 19 69-77

Westerweel J 1993 Digital particle image velocimetry - theory and application PhD Thesis Technical University of Delft

Westerweel J, Dabiri D and Gharib M 1997 The effects of a discrete window offset on the accuracy of cross correlation analysis digital PIV recording Exp. Fluids 23 20-8

Willert C 1992 The interaction of modulated vortex pairs with a free surface $P h D$ Thesis University of California, San Diego

Willert C and Gharib M 1991 Digital particle image velocimetry Exp. Fluids 10 181-93 